\title{
An SVD based strategy for receding horizon control of input constrained linear systems
}

\author{
Osvaldo J. Rojas*, Graham C. Goodwin*, María M. Serón* and \\ Arie Feuer ${ }^{\diamond}$ \\ ${ }^{*}$ School of Electrical Engineering \& Computer Science \\ The University of Newcastle, Australia. \\ $\diamond$ Dept. of Electrical Engineering, Technion, Israel.
}

\begin{abstract}
A sub-optimal receding horizon control strategy for input constrained linear systems is presented. The strategy is based on a singular value decomposition (SVD) of the Hessian of the quadratic performance index generally considered in Model Predictive Control (MPC). The singular vectors are employed to generate a basis function expansion of the unconstrained solution to the finite horizon optimal control problem. At each sampling time, a feasible control sequence is determined by selecting a variable subset of the basis representation. No solution to the associated quadratic program is needed. For cases in which the Hessian is poorly conditioned, the proposed strategy can provide a sub-optimal solution with minimal performance degradation. Properties of the singular values of the Hessian are also studied: it is shown that, for sufficiently long prediction horizons, the singular values are arbitrarily close to the magnitude of the energy density spectrum of the system seen by the performance index.
\end{abstract}

\section{Introduction}

Model predictive control (MPC) has become a standard approach in advanced control of constrained multivariable systems (Qin and Badgwell, 2003; Mayne et al., 2000). MPC solves, at each sampling time, a fi nite horizon constrained optimization problem based on the current value of the state vector. Only the fi rst move of the resulting optimal control sequence is applied to the system. At the next time step, when a new state measurement is available, the optimization procedure is repeated over a shifted horizon. The resulting control strategy leads to a receding horizon control (RHC) policy.

One of the key advantages of MPC is its ability to incorporate constraints directly into the problem formulation. When a quadratic cost is considered, the fi nite horizon 
optimal control problem can be cast into a quadratic program (QP) for which effi cient numerical algorithms are available - see, for example, (Bartlett et al., 2002; Rao et al., 1998). However, one important diffi culty associated with this class of algorithms is that, depending upon the specifi c application, considerable computational effort may still be required. This, in turn, has limited the application of MPC to relatively "slow" or "small" dimensional systems.

Some important research efforts have recently been reported aimed at reducing the online computational burden associated with the solution of QP problems. Independently Serón et al. (2000) and Bemporad et al. (2002) have provided a fi nitely parameterized explicit solution to QP problems. The solution is characterized by a partition of the state space into a fi nite number of regions in which the corresponding control law is an affi ne function of the states. The main advantage of this approach is that the explicit QP solution can be computed entirely off-line. Only a simple search to determine the region that contains the current state is needed on-line. However, a potential disadvantage of this approach is that the state partition becomes increasingly more complex as the prediction horizon increases.

An alternative approach to reducing the on-line computational effort is the development of some sort of sub-optimal solution to the QP problem. In this case, an appropriate balance between loss of performance and improved computational speed needs to be considered. This method has been proposed by several authors - see, for example, (Johansen et al., 2002; Kouvaritakis et al., 2002).

In this contribution, we propose a sub-optimal receding horizon control strategy, based on a singular value decomposition (SVD) of the Hessian of the quadratic performance index generally minimized in Model Predictive Control. The SVD of the Hessian allows one to highlight the components of the decision variable in the optimization problem that have greater influence on the performance index. Furthermore, the singular vectors of the Hessian provide a set of orthogonal basis vectors spanning the control space. At each sampling time, the set of singular vectors are used to construct a basis function expansion of the unconstrained optimal control solution to the optimization problem. By considering a variable subset of the basis representation, the proposed SVD- RHC strategy ensures that no constraints are violated at any time.

In this paper we focus on the input constraint case only. This simplifying assumption ensures that a feasible solution to the fi nite horizon optimization problem can always be found. With this set-up the proposed receding horizon control strategy is especially simple to implement. In the more general case in which state and input constraints are present, the strategy requires the computation of a feasible solution fi rst. This, in turn, could be achieved with a separate linear programming problem but distracts from the simplicity of the method.

The strategy proposed in this paper has evolved from ideas fi rst developed by the authors for anti-windup control in cross-directional (CD) control systems (Rojas et al., 2002a; Rojas et al., 2002b). CD systems are typically high dimensional multivariable problems (with up to 300 actuator/sensor pairs) characterized by strong spatial interaction among actuators. This coupling leads to poor conditioning of the steady state interaction 
matrix describing the system.

For many ill-conditioned problems, the singular value approach appears to be an effective way of characterizing the elements of the problem in terms of the diffi culty associated with handling them. Large singular values indicate a large gain between the corresponding control basis vector and the cost function. This suggests that a near optimal strategy would be to construct a feasible control vector by retaining the basis vectors associated with the largest singular values while progressively discarding the components associated with the smallest singular values until a constraint boundary is reached.

Parallel work relating to a similar strategy has been described in (Sanchis et al., 2001). Also, note that there is a link between the algorithm described here and recent work reported in (Kojima and Morari, 2001) where a fixed set of singular values is used to simplify the continuous MPC problem. By way of contrast, we use a variable number of singular values at each time step.

The layout of the remainder of the paper is as follows: in Section 2 we provide a brief review of quadratic receding horizon control. In Section 3 we analyse the singular value decomposition of the Hessian of the performance index and describe the proposed receding horizon strategy in Section 4. The stability of the closed loop is studied in Section 5. We draw some connections between the singular values of the Hessian and the frequency response of the system in Section 6 and fi nally we present a simulation example based on a 3 input - 3 output plant in Section 7.

A preliminary version of this paper was presented in (Rojas et al., 2003).

\section{Review of quadratic constrained receding horizon con- trol}

Consider a discrete time linear system described in state space form by

$$
\begin{aligned}
& x_{k+1}=A x_{k}+B u_{k}, \quad k=0,1, \ldots \\
& y_{k}=C x_{k}
\end{aligned}
$$

where $x_{k} \in \mathbb{R}^{n}$ is the state vector, $u_{k} \in \mathbb{R}^{m}$ is the input vector, and $y_{k} \in \mathbb{R}^{p}$ is the output vector. Assume that the pair $(A, B)$ is stabilisable and that the system is subject to input constraints

$$
u_{k+t} \in \mathbb{U}, \quad t=0,1, \ldots .
$$

The input constraint set $\mathbb{U}$ is a convex, compact subset of $\mathbb{R}^{m}$ which contains the origin in its interior (Mayne et al., 2000).

We are interested in the quadratic performance index

$$
J_{N}\left(x_{k}, \mathbf{u}\right)=\sum_{t=0}^{N-1}\left[x_{k+t \mid k}^{T} Q x_{k+t \mid k}+u_{k+t}^{T} R u_{k+t}\right]+x_{k+N \mid k}^{T} P x_{k+N \mid k},
$$


where the vector $\mathbf{u}$ contains the control moves $u_{k+t}$ defi ned over the fi nite horizon $t \in[0, N-1]$ with $N \in \mathbb{Z}^{+}$and $N \geq 1$, that is

$$
\mathbf{u}=\left[\begin{array}{llll}
u_{k}^{T} & u_{k+1}^{T} & \cdots & u_{k+N-1}^{T}
\end{array}\right]^{T} .
$$

The weighting matrices $Q$ and $R$ satisfy

$$
\begin{aligned}
& Q=Q^{T}>0, \\
& R=R^{T}>0,
\end{aligned}
$$

whilst the terminal weighting matrix $P$ is the unique symmetric positive defi nite solution of the discrete time algebraic Riccati equation

$$
P=Q+A^{T} P A-K^{T}\left(R+B^{T} P B\right) K
$$

where

$$
\begin{aligned}
& K=\bar{R}^{-1} B^{T} P A, \\
& \bar{R}=R+B^{T} P B .
\end{aligned}
$$

The vector $x_{k+t \mid k}$ in (3) represents the prediction of the state vector of the system at time $k+t$ given the information available at time $k$ and based on the model in (1) i.e.,

$$
\begin{aligned}
& x_{k+t+1 \mid k}=A x_{k+t \mid k}+B u_{k+t}, \quad t=0,1, \ldots \\
& \text { and } x_{k \mid k}=x_{k} .
\end{aligned}
$$

Model Predictive Control (MPC) solves, at each time step, the following fi nite horizon optimal control problem

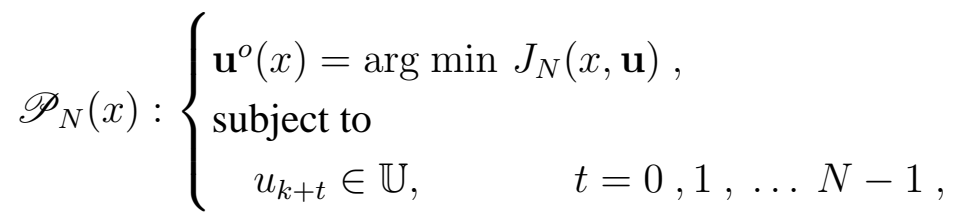

where $x=x_{k}$ is the state measurement at the current time $k$. Only the first move of the optimal control vector $\mathbf{u}^{o}(x)$ is applied to the system (1), i.e., $u_{k}=u_{k}^{o}(x)$. The optimization procedure is repeated at each time step when a new state measurement $x$ becomes available and over a shifted horizon. The resulting control strategy leads to a receding horizon control (RHC) policy.

\section{Singular value decomposition of the optimization prob- lem}

In this section, we analyse in more detail the structure of the performance index $J_{N}(x, \mathbf{u})$ in (3). Our main objective is to gain insight into the inherent structure of $J_{N}(x, \mathbf{u})$ so 
as to set the background for the sub-optimal receding horizon control strategy we will introduce in Section 4.

Using matrix notation, $J_{N}(x, \mathbf{u})$ can be expressed as follows:

$$
J_{N}(x, \mathbf{u})=x^{T} Y x+\mathbf{u}^{T} H \mathbf{u}+2 \mathbf{u}^{T} F x,
$$

where

$$
\begin{aligned}
& Y=Q+\Lambda^{T} \mathbf{Q} \Lambda \quad \in \mathbb{R}^{n \times n}, \\
& H=\mathbf{R}+\Gamma^{T} \mathbf{Q} \Gamma \quad \in \mathbb{R}^{N m \times N m} \\
& F=\Gamma^{T} \mathbf{Q} \Lambda \quad \in \mathbb{R}^{N m \times n} .
\end{aligned}
$$

Also

$$
\begin{aligned}
& \mathbf{Q}=\operatorname{diag}\{Q, \ldots, Q, P\} \\
& \mathbf{R}=\operatorname{diag}\{R, \ldots, R\}
\end{aligned}
$$

and

$$
\Lambda=\left[\begin{array}{c}
A \\
A^{2} \\
\vdots \\
A^{N}
\end{array}\right], \Gamma=\left[\begin{array}{cccc}
B & 0 & \cdots & 0 \\
A B & B & \cdots & 0 \\
\vdots & \vdots & \ddots & \vdots \\
A^{N-1} B & A^{N-2} B & \cdots & B
\end{array}\right]
$$

Matrices $\Lambda$ and $\Gamma$ defi ne the relationship between the vector of control moves $\mathbf{u}$ and the vector

$$
\mathbf{x}=\left[\begin{array}{llll}
x_{k+1 \mid k}^{T} & x_{k+2 \mid k}^{T} & \cdots & x_{k+N \mid k}^{T}
\end{array}\right]^{T}
$$

which contains the future time evolution of the state vector of system (1) i.e.,

$$
\mathbf{x}=\Lambda x+\Gamma \mathbf{u} .
$$

The unconstrained solution to the fi nite horizon optimal control problem $\mathscr{P}_{N}(x)$ is readily found to be

$$
\mathbf{u}_{u c}^{o}(x)=-H^{-1} F x .
$$

However, there is no such simple explicit expression for the constrained optimal solution to $\mathscr{P}_{N}(x)$. Indeed, as pointed out by Bemporad et al. (2002) and Serón et al. (2000) an explicit solution for $\mathbf{u}^{o}(x)$ in (9) consists of a partition of the state space into a fi nite (but possibly large) number of regions in which the corresponding control law is an affi ne function of the states.

In this paper, we explore an alternative approach. We fi rst seek to determine which of the components of the vector of control moves $u$ have the biggest impact on the reduction of the magnitude of the performance index $J_{N}(x, \mathbf{u})$. Note that this is determined by the structure of $J_{N}(x, \mathbf{u})$ in terms of the control vector $\mathbf{u}$ and, hence, is independent of the current value of the state vector $x$. To clarify this, we consider a singular value decomposition (SVD) (Golub and Van Loan, 1996) of the Hessian $H$ of $J_{N}(x, \mathbf{u}$ ),

$$
H=V S V^{T} .
$$


The matrix $S \in \mathbb{R}^{N m \times N m}$ is diagonal, with positive values $\left\{\sigma_{1}, \sigma_{2}, \ldots \sigma_{N m}\right\}$ arranged in decreasing order. The scalars $\left\{\sigma_{i}\right\}_{i=1}^{N m}$ are the singular values of $H$. The columns of the matrix $V \in \mathbb{R}^{N m \times N m}$ contain the right singular vectors of $H$; however, in this case, left and right singular vectors are identical since $H$ is symmetric - see (12). An important property is that the singular vector matrix $V$ is orthogonal i.e., $V^{T} V=V V^{T}=I_{N m}$, where $I_{N m}$ is the $N m$ by $N m$ identity matrix. This, in turn, implies that the columns of $V$ provide a set of orthogonal basis vectors spanning $\mathbb{R}^{N m}$. As a result, the vector of control moves $\mathbf{u}$ in (4) can be expressed as a linear combination of the singular vectors of $H$,

$$
\mathbf{u}=V \widetilde{\mathbf{u}}=\sum_{i=1}^{N m} \mathbf{v}_{i} \widetilde{u}_{i},
$$

where $\mathbf{v}_{i}, i \in \mathcal{I}_{N m} \triangleq\{1,2, \ldots, N m\}$, are the columns of $V$ and $\widetilde{u}_{i}$ are the entries of the vector $\widetilde{\mathbf{u}}$.

Using the change of variable defi ned in (16), we can next recast the performance index (10) in terms of the new vector of control moves $\widetilde{\mathbf{u}}$,

$$
\widetilde{J}_{N}(x, \widetilde{\mathbf{u}}) \triangleq J_{N}(x, V \widetilde{\mathbf{u}})=x^{T} Y x+\widetilde{\mathbf{u}}^{T} S \widetilde{\mathbf{u}}+2 \widetilde{\mathbf{u}}^{T} V^{T} F x
$$

Completing squares around the unconstrained optimal solution

$$
\widetilde{\mathbf{u}}_{u c}^{o}(x)=-S^{-1} V^{T} F x,
$$

we fi nally obtain

$$
\begin{aligned}
\widetilde{J}_{N}(x, \widetilde{\mathbf{u}}) & =\widetilde{J}_{N, u c}^{o}(x)+\left(\widetilde{\mathbf{u}}-\widetilde{\mathbf{u}}_{u c}^{o}(x)\right)^{T} S\left(\widetilde{\mathbf{u}}-\widetilde{\mathbf{u}}_{u c}^{o}(x)\right)= \\
& =\widetilde{J}_{N, u c}^{o}(x)+\sum_{i=1}^{N m} \sigma_{i}\left(\widetilde{u}_{i}-\widetilde{u}_{i, u c}^{o}\right)^{2},
\end{aligned}
$$

where $\widetilde{u}_{i, u c}^{o}$ is the $i$-th entry of vector $\widetilde{\mathbf{u}}_{u c}^{o}(x)$, and $\widetilde{J}_{N, u c}^{o}(x)$ is the unconstrained value function. Clearly, whenever $\widetilde{\mathbf{u}}=\widetilde{\mathbf{u}}_{u c}^{o}(x)$ we have that $\widetilde{J}_{N}\left(x, \widetilde{\mathbf{u}}_{u c}^{o}\right)=\widetilde{J}_{N, u c}^{o}(x)$. Eq. (18) shows that, in the singular value space of $H$, the entries of $\widetilde{\mathbf{u}}$ are ordered starting from the one that influences the performance index the most and ending with the one that influences the performance index the least, since $\sigma_{1} \geq \sigma_{2} \geq \ldots \geq \sigma_{N m}$.

In the next Section we will propose an algorithm able to fi nd a sub-optimal solution to the constrained optimization problem $\mathscr{P}_{N}(x)$, based on the insight provided by the SVD of the Hessian $H$. The intuitive rationale behind our approach is that a sub-optimal feasible solution to $\mathscr{P}_{N}(x)$ should be constructed by considering the components of $\widetilde{\mathbf{u}}$ that are more cost effective i.e., those components associated with largest singular values.

Remark 3.1. Note that the SVD of the Hessian $H$ adopted here is also a useful approach to deal with the common issue of ill-conditioning of $H$ when long horizons $N$ are used. This is the consequence of the entries of $\Gamma$ in (14) being related to powers of the state matrix $A$. The conditioning of $H$ becomes especially sensitive to the prediction horizon $N$ when the 
system in (1) is open-loop unstable. In this case, the difference between $A$ and $A^{N-1}$ can be signifi cant even for short horizons $N$. Rossiter et al. (1998) have noted this diffi culty and have proposed to pre-stabilize the prediction model (8) by re-parameterizing the input as $u_{k}=-L x_{k}+r_{k}$ where $L$ is any linear stabilizing gain for $(A, B)$. Alternatively, one could improve the conditioning of the problem by simply discarding the singular values of $H$ below certain value and solve the QP problem with the modifi ed performance index.

Remark 3.2. A numerical problem related to that described in Remark 3.1 is that of the poor-conditioning of the system model (1). This can occur when the gain of the model is highly dependent on the direction of the input. An example in which this diffi culty appears is the cross-directional control of web forming processes (Featherstone et al., 2000). In these cases, poor-conditioning is quantifi ed via a SVD of the system model or, equivalently, of the matrix $\Gamma$ in (14). Singular values smaller than a certain pre-specifi ed threshold are discarded and a process model with a lower condition number is re-derived and used to implement the controller. This approach is usually known as singular value thresholding (Qin and Badgwell, 2003; Aoyama et al., 1997). It is not diffi cult to see that there exists a direct connection between the singular values of the matrix $\Gamma$ and the singular values of $H$. To clarify, consider the following SVD

$$
\Gamma=U \bar{S} V^{T},
$$

and for simplicity consider $R=0$ and $Q=I_{n}$ with $I_{n}$ the $n$ by $n$ identity matrix. Then, from the defi nition of $H$ in (12) we obtain

$$
H=\Gamma^{T} \Gamma=V \bar{S}^{2} V^{T},
$$

since $U^{T} U=U U^{T}=I_{N n}$.

The connection between the singular values of $H$ and the open loop properties of the system will be further investigated in Section 6.

\section{Receding horizon strategy using singular value decom- position}

We next seek a modifi cation of the unconstrained solution $\widetilde{\mathbf{u}}_{u c}^{o}$ in (17) in order to construct a vector of control moves $\widetilde{\mathbf{u}}$ that satisfi es the constraints imposed on the fi nite horizon optimal control problem $\mathscr{P}_{N}(x)$, without incurring the full computational load of solving the associated QP problem.

The key concept underpinning the approach suggested in this contribution is based on the representation of the performance index $\widetilde{J}_{N}(x, \widetilde{\mathbf{u}})$ in $(18)$. If one had to construct a sub-optimal solution to $\mathscr{P}_{N}(x)$ based on the selection of a subset of the elements of $\widetilde{\mathbf{u}}_{u c}^{o}$, then it would be reasonable to include, in the selection, those components of $\widetilde{\mathbf{u}}_{u c}^{o}$ with the biggest impact on the reduction of the magnitude of $\widetilde{J}_{N}(x, \widetilde{\mathbf{u}})$ i.e., those associated with the biggest singular values. Moreover, the components of $\widetilde{\mathbf{u}}_{u c}^{o}$ associated with the 
smallest singular values are more likely to violate the input constraints in (2), since they are inversely proportional to the value of $\sigma_{i}$ - see (17).

The above suggests that a feasible solution to the constrained optimization problem $\mathscr{P}_{N}(x)$ can be constructed by considering the vector $\widetilde{\mathbf{u}}_{u c}^{o}$ and progressively discarding its components, starting from the one associated with the smallest singular value, until the resulting control vector is brought inside the constraint set. Therefore, if the unconstrained solution to $\mathscr{P}_{N}(x)$ is

$$
\mathbf{u}_{u c}^{o}(x)=V \widetilde{\mathbf{u}}_{u c}^{o}(x)=\sum_{i=1}^{N m} \mathbf{v}_{i} \widetilde{u}_{i, u c}^{o}(x)
$$

then we can defi ne

$$
\mathbf{u}_{s v d}(x)=\sum_{i=1}^{r} \mathbf{v}_{i} \widetilde{u}_{i, u c}^{o}(x)+\left(\mathbf{v}_{r+1} \alpha \widetilde{u}_{r+1, u c}^{o}(x)\right)
$$

for a given integer $r \in \mathcal{I}_{N m} \triangleq\{1,2, \ldots, N m\}$ and a given scaling factor $\alpha \in[0,1)$. It is clear that if $r=N m$, then $\alpha=0$ and the unconstrained solution (19) is recovered.

The proposed singular value based receding horizon control strategy (SVD- RHC) is described in the following algorithm.

\section{Algorithm 4.1 (SVD- RHC strategy).}

1. At time $k$, given the current state $x_{k}$ calculate the unconstrained optimal solution to $\mathscr{P}_{N}\left(x_{k}\right)$ i.e.,

$$
\widetilde{\mathbf{u}}_{u c}^{o}\left(x_{k}\right)=-S^{-1} V^{T} F x_{k} .
$$

2. Find the largest $\gamma \triangleq r+\alpha$, where $r \in \mathcal{I}_{N m} \triangleq\{1,2, \ldots, N m\}$ and $\alpha \in[0,1)$, such that the vector

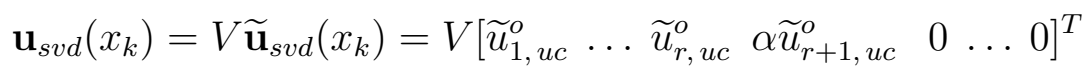

lies on the boundary of the constraint set in $\mathbb{R}^{N m}$ constructed from the input constraint set $\mathbb{U}$.

3. Apply, as the current control move, the first $m$ elements of the control vector $\mathbf{u}_{s v d}\left(x_{k}\right)$ i.e.,

$$
u_{k}=D \mathbf{u}_{s v d}\left(x_{k}\right),
$$

where

$$
D=\left[\begin{array}{ll}
I_{m} & \mathbf{0}_{m \times(N-1) m}
\end{array}\right],
$$

and $\mathbf{0}_{m \times(N-1) m}$ is a sub-matrix of specifi ed dimensions with zero entries.

4. Set $k=k+1$ and return to step 1 . 


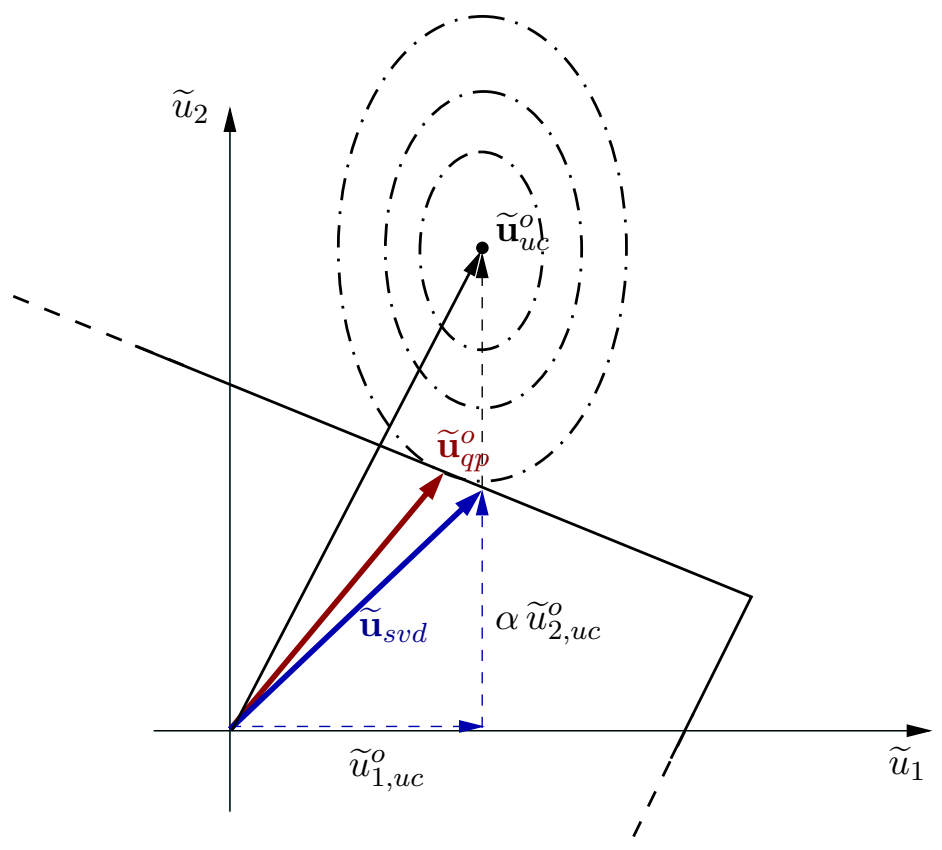

Figure 1: Graphical comparison between the optimal QP solution $\widetilde{\mathbf{u}}_{q p}^{o}$ to $\mathscr{P}_{N}(x)$ and the solution $\widetilde{\mathbf{u}}_{s v d}$ obtained with the SVD- RHC strategy for a simple case with $N m=2$.

Note that the proposed SVD- RHC strategy computes, at each time step, a different value of $\gamma$ such that the resulting control vector $\mathbf{u}_{s v d}(x)$ is feasible. In addition, the strategy ensures that the available control authority is used in full, by selecting the largest possible $\gamma \triangleq r+\alpha$ so that $\mathbf{u}_{s v d}(x)$ is on the boundary of the constraint set.

The control law defi ned by the SVD- RHC strategy can be expressed as a state dependent feedback gain matrix $K_{\gamma}\left(x_{k}\right)$ such that

$$
u_{k}=-K_{\gamma}\left(x_{k}\right) x_{k},
$$

with $K_{\gamma}\left(x_{k}\right)$ given by

$$
K_{\gamma}\left(x_{k}\right)=D V \operatorname{diag}(\underbrace{1, \ldots, 1}_{r}, \alpha, \underbrace{0, \ldots, 0}_{N m-r-1}) S^{-1} V^{T} F .
$$

Remark 4.1. The control vector $\mathbf{u}_{s v d}(x)$ is clearly a sub-optimal solution to $\mathscr{P}_{N}(x)$ in (9). However, for cases in which the Hessian of the performance index (10) is poorlyconditioned, the proposed strategy is expected to deliver a feasible solution which is close to the QP optimal solution. Indeed, when $H$ is ill-conditioned there will be only a few relevant singular values $\sigma_{i}$, while all the others may be discarded without excessively increasing the value of the performance index. A graphical interpretation of the SVD- RHC strategy is shown in Fig. 1 for a simple case in which $N m=2$. The control vectors are plotted in the singular value space of $H$. The ellipsoids represents the level curves of the performance index. The point of tangency between the largest level set and the constraint polytope represents the optimal solution $\widetilde{\mathbf{u}}_{q p}^{o}(x)$ obtained by solving the QP problem. By 
way of contrast, the solution $\widetilde{\mathbf{u}}_{s v d}(x)$ delivered by the proposed strategy is constructed by keeping the fi rst component of $\widetilde{\mathbf{u}}_{u c}^{o}(x)$ entirely and scaling the second component so that the constraints are met. We observe from Fig. 1 that the solution $\widetilde{\mathbf{u}}_{s v d}(x)$ is very close to the solution $\widetilde{\mathbf{u}}_{q p}^{o}(x)$ in this case.

Remark 4.2. As mentioned in Section 1, a special case of the SVD- RHC strategy has previously been used in the context of the cross-directional control of web forming processes (Rojas et al., 2002a). In this latter work, only the singular values of the static interaction matrix of the system were considered. However, in the light of the formulation given above we can provide a more general interpretation of the anti-windup algorithm in (Rojas et al., 2002a): the cross-directional control objective can be cast into a simplifi ed version of the quadratic performance index (10) in which the prediction horizon is $N=1$ and only the spatial interaction between actuators determines the effect of the control sequence $\mathbf{u}$ over the performance index $J_{N}(x, \mathbf{u})$. This is an example of an application of the strategy described here in which $\mathbf{u}$ shows high spatial dimensionality but only one component in the time dimension.

\section{Stability analysis}

We next analyse the stability properties of the SVD- RHC strategy by means of simple Lyapunov arguments. First, we require the following Lemma.

Lemma 5.1. Consider the matrices

$$
\begin{aligned}
& \bar{K} \triangleq H^{-1} F, \\
& \bar{K}_{\gamma} \triangleq V \operatorname{diag}(1, \ldots, 1, \alpha, 0, \ldots, 0) S^{-1} V^{T} F, \quad \gamma \in[0, N m],
\end{aligned}
$$

where $H$ and $F$ are defi ned in (12) and (13) respectively and $S$ and $V$ are defi ned in (15). Let

$$
\bar{E}_{\gamma} \triangleq \bar{K}-\bar{K}_{\gamma}
$$

Then, given any two values $\gamma_{1}, \gamma_{2} \in[0, N m]$ such that $\gamma_{1} \geq \gamma_{2}$, we have that

$$
\left\|\bar{E}_{\gamma_{1}}\right\|_{2} \leq\left\|\bar{E}_{\gamma_{2}}\right\|_{2}
$$

Proof. We fi rst note that

$$
\begin{aligned}
& \mathbf{u}_{u c}^{o}(x)=-\bar{K} x \\
& \mathbf{u}_{s v d}(x)=-\bar{K}_{\gamma} x .
\end{aligned}
$$

Replacing (15) in (23) we can write

$$
\bar{K}=V S^{-1} V^{T} F .
$$

Thus

$$
\bar{E}_{\gamma} \triangleq \bar{K}-\bar{K}_{\gamma}=V \operatorname{diag}(0, \ldots, 0,1-\alpha, 1, \ldots, 1) S^{-1} V^{T} F
$$


Next, let $d_{\gamma}=\bar{E}_{\gamma} x$ for any given state vector $x \in \mathbb{R}^{n}$. Then we have

$$
\begin{aligned}
\left\|d_{\gamma}\right\|_{2} & =\left\|V \operatorname{diag}(0, \ldots, 0,1-\alpha, 1, \ldots, 1) S^{-1} V^{T} F x\right\|_{2} \\
& =\left\|\operatorname{diag}(0, \ldots, 0,1-\alpha, 1, \ldots, 1) S^{-1} V^{T} F x\right\|_{2},
\end{aligned}
$$

since $V$ is orthogonal. We can see that, as the value of $\gamma \triangleq r+\alpha$ is increased, there are fewer components of the vector $S^{-1} V^{T} F x$ that are considered when constructing $d_{\gamma}$. Hence, given $\gamma_{1}, \gamma_{2} \in[0, N m]$ such that $\gamma_{1} \geq \gamma_{2}$, we can conclude that

$$
\left\|d_{\gamma_{1}}\right\|_{2} \leq\left\|d_{\gamma_{2}}\right\|_{2} \Longleftrightarrow\left\|\bar{E}_{\gamma_{1}} x\right\|_{2} \leq\left\|\bar{E}_{\gamma_{2}} x\right\|_{2}, \quad \forall x \in \mathbb{R}^{n} .
$$

We can now choose $x=v_{1}$ where $v_{1}$ is the right singular vector associated with the largest singular value of the matrix $\bar{E}_{\gamma_{1}}$. In this case

$$
\left\|\bar{E}_{\gamma_{1}} v_{1}\right\|_{2}=\left\|\bar{E}_{\gamma_{1}}\right\|_{2}\left\|u_{1}\right\|_{2}=\left\|\bar{E}_{\gamma_{1}}\right\|_{2}
$$

where $u_{1}$ is the corresponding left singular vector. Recall that both $u_{1}$ and $v_{1}$ are vectors with norm one. Finally, replacing (27) in (26) we obtain

$$
\begin{aligned}
& \left\|\bar{E}_{\gamma_{1}}\right\|_{2} \leq\left\|\bar{E}_{\gamma_{2}} v_{1}\right\|_{2} \leq\left\|\bar{E}_{\gamma_{2}}\right\|_{2}\left\|v_{1}\right\|_{2}=\left\|\bar{E}_{\gamma_{2}}\right\|_{2} \\
& \Longrightarrow\left\|\bar{E}_{\gamma_{1}}\right\|_{2} \leq\left\|\bar{E}_{\gamma_{2}}\right\|_{2} .
\end{aligned}
$$

This completes the Proof.

Corollary 5.2. The norm of the matrix $\bar{E}_{\gamma} \triangleq \bar{K}-\bar{K}_{\gamma}$ satisfi es

$$
0 \leq\left\|\bar{E}_{\gamma}\right\|_{2} \leq\|\bar{K}\|_{2}, \quad \forall \gamma \in[0, N m] .
$$

Proof. Directly from Lemma 5.1 with $\gamma_{2}=0$. Also, note that $\left\|\bar{E}_{\gamma}\right\|_{2}=0$ when $\gamma=N m$ by construction.

We are now able to present the main stability result for the SVD- RHC strategy.

Theorem 5.3. Consider the discrete time linear system (1) and the SVD- RHC control law $u_{k}=-K_{\gamma} x_{k}$ determined via Algorithm 4.1. Consider $\delta>0$ and $\gamma^{*} \in[0, N m]$ such that

$$
\left\|\bar{E}_{\gamma^{*}}\right\|_{2}<\sqrt{\frac{\lambda_{\min }(Q)-\delta \lambda_{\max }(P)}{\|\bar{R}\|_{2}}},
$$

where $P$ is the solution to the discrete time algebraic Riccati equation in (5) and $\bar{R}$ is defi ned in (7). Then the closed loop system is exponentially stable for all $\gamma \geq \gamma^{*}$.

Proof. The closed-loop system obtained by applying the SVD- RHC strategy to the system (1) is readily seen to be

$$
x_{k+1}=A x_{k}-B K_{\gamma} x_{k}=A_{K} x_{k}+B E_{\gamma} x_{k},
$$


where the matrix $A_{K}=A-B K$ is the closed loop state matrix obtained using the unconstrained optimal LQR solution $u_{k}=-K x_{k}$, with $K$ as in (6), and

$$
E_{\gamma} \triangleq D \bar{E}_{\gamma}=K-K_{\gamma}
$$

where matrices $D$ and $K_{\gamma}$ are defi ned in (21) and (22) respectively. Next, consider the Lyapunov function candidate $\mathscr{V}(x)=x^{T} P x$, with $P$ being the symmetric positive definite solution to the discrete time algebraic Riccati equation (5). Along the closed-loop trajectories in (28) we have

$$
\begin{array}{r}
\Delta \mathscr{V}\left(x_{k}\right)=\mathscr{V}\left(x_{k+1}\right)-\mathscr{V}\left(x_{k}\right)=x_{k}^{T}\left\{-Q-K^{T} R K+E_{\gamma}^{T} B^{T} P B E_{\gamma}+\right. \\
\left.E_{\gamma}^{T} B^{T} P A_{K}+A_{K}^{T} P B E_{\gamma}\right\} x_{k} .
\end{array}
$$

Using $B^{T} P B=\bar{R}-R$ and $B^{T} P A_{K}=R K$ and after some algebraic manipulation we obtain

$$
\Delta \mathscr{V}\left(x_{k}\right)=-x_{k}^{T}\left\{Q+\left(K-E_{\gamma}\right)^{T} R\left(K-E_{\gamma}\right)-E_{\gamma}^{T} \bar{R} E_{\gamma}\right\} x_{k} .
$$

Since the second term in (29) is negative semidefi nite, we can write

$$
\Delta \mathscr{V}\left(x_{k}\right) \leq-x_{k}^{T}\left\{Q-E_{\gamma}^{T} \bar{R} E_{\gamma}\right\} x_{k} .
$$

Given $\delta>0$ we seek to determine a suffi cient condition on $\bar{E}_{\gamma}$ such that the inequality

$$
Q-E_{\gamma}^{T} \bar{R} E_{\gamma} \geq \delta P
$$

holds. This condition is readily seen to be

$$
\left\|E_{\gamma}\right\|_{2} \leq\|D\|_{2}\left\|\bar{E}_{\gamma}\right\|_{2}<\sqrt{\frac{\lambda_{\min }(Q)-\delta \lambda_{\max }(P)}{\|\bar{R}\|_{2}}},
$$

since $\|D\|_{2}=1$. Clearly $\delta$ needs to be selected such that $\lambda_{\min }(Q)>\delta \lambda_{\max }(P)$.

The result of Corollary 5.2 and the continuity of the matrix 2-norm guarantee that there exists $\gamma^{*} \in[0, N m]$ such that (30) holds. In addition, Lemma 5.1 ensures that (30) holds for all $\gamma \geq \gamma^{*}$. Therefore, since $\mathscr{V}(x)=x^{T} P x$ and $\Delta \mathscr{V}\left(x_{k}\right) \leq-\delta x^{T} P x$, exponential stability of the closed loop system results if $\gamma \geq \gamma^{*}$. This completes the Proof.

The result of Theorem 5.3 provides a suffi cient condition for the exponential stability of the SVD- RHC strategy. We next seek to estimate the region of attraction of the SVDRHC strategy. Let us fi rst introduce the following defi nition.

Definition 5.4 (Region of admissible states $C_{\gamma^{*}}$ ).

Consider the value $\gamma^{*} \in[0, N m]$ defi ned in Theorem 5.3. Let $C_{\gamma^{*}}$ represent the set of all states $x \in \mathbb{R}^{n}$ for which the SVD- RHC strategy of Algorithm 4.1 maps $x$ into $\gamma$ such that $\gamma \geq \gamma^{*}$. That is

$$
C_{\gamma^{*}} \triangleq\left\{x \in \mathbb{R}^{n} \mid \mathscr{M}_{\text {svd }}(x) \geq \gamma^{*}\right\}
$$

where $\mathscr{M}_{s v d}(x): x \longrightarrow \gamma$ is the mapping implicitly defi ned in Algorithm 4.1. 
The region $C_{\gamma^{*}}$ defi nes the state space region in which the stability condition of Theorem 5.3 holds. In order to obtain an estimate of the region of attraction of the SVD-RHC strategy we need to fi nd a positively control invariant set for the control law $u_{k}=-K_{\gamma} x_{k}$ that is contained in $C_{\gamma^{*}}$ (Blanchini, 1999). One possibility is to use ellipsoidal sets, in particular, the level sets of the Lyapunov function $\mathscr{V}(x)=x^{T} P x$ employed in the proof of Theorem 5.3.

Hence, let us select the largest $\rho>0$ such that the ellipsoidal region

$$
\Omega \triangleq\left\{x \in \mathbb{R}^{n} \mid x^{T} P x \leq \rho\right\}
$$

is completely contained in the region $C_{\gamma^{*}}$. Then, the SVD- RHC algorithm guarantees that all states in $\Omega$ yield $\gamma \geq \gamma^{*}$, and hence all trajectories starting in $\Omega$ will converge exponentially to the origin of the state space. Note that $\rho$ can be determined completely off - line since it depends solely on the value $\gamma^{*}$ provided by the result of Theorem 5.3 and the corresponding region $C_{\gamma^{*}}$.

Remark 5.1. The fact that the input constraint set $\mathbb{U}$ in (2) is non empty implies that the region $C_{\gamma^{*}}$ of Defi nition 5.4 is also non empty. Indeed, let $C_{N m}$ be the state space region in which no constraints are active, that is

$$
C_{N m} \triangleq\left\{x \in \mathbb{R}^{n} \mid \mathscr{M}_{s v d}(x)=N m\right\} .
$$

Clearly the region $C_{N m}$ is non empty since $\exists x \in \mathbb{R}^{n}$ such that $-K x \in \mathbb{U}$, in particular $x=0$. However, based on Defi nition 5.4 we have that

$$
C_{N m} \subseteq C_{\gamma^{*}}, \quad \forall \gamma^{*} \in[0, N m]
$$

from which we conclude that the region $C_{\gamma^{*}}$ is non empty.

\section{Asymptotic behaviour of singular values}

In this section we study the asymptotic behaviour of the singular values of the Hessian $H$ for long prediction horizons $N$. The purpose of this analysis is to gain a better understanding of the inherent structure of the performance index $J_{N}(x, \mathbf{u})$ for long horizons $N$. In addition, we believe that the result presented here shows that the SVD- RHC strategy described in Section 4 can be especially effective when long prediction horizons are used. We consider the case of the general Hessian $H$. However, for simplicity of exposition, we restrict the model of the system (1) to be SISO and open-loop stable. Extensions to MIMO and unstable models are discussed elsewhere - see (Rojas and Goodwin, 2003).

Consider the fi nite horizon optimal control problem $\mathscr{P}_{N}(x)$ in (9) with a performance index $J_{N}(x, \mathbf{u})$ as per (3) and fi nal state weighting $P=Q$ (note that this choice is not restrictive since we are interested in the case $N \rightarrow \infty$ ). Also, consider

$$
Q=C^{T} C, \text { and } R=\lambda>0
$$


i.e., we are interested in regulating the output $y_{k}$ of the system to zero. With this set-up we can defi ne

$$
\bar{\Gamma}=\left(I_{N} \otimes C\right) \Gamma,
$$

with $\otimes$ the usual Kronecker product and $I_{N}$ the $N$ by $N$ identity matrix. This defi nition leads to the following expression for the Hessian $H$ in (12)

$$
H=\bar{\Gamma}^{T} \bar{\Gamma}+\lambda I_{N}
$$

Next, consider the impulse response $\left\{h_{k}\right\}_{k=0}^{\infty}$ of the discrete time linear system (1) i.e.,

$$
\left\{h_{k}\right\}_{k=0}^{\infty}=\left\{0, C B, C A B, C A^{2} B, \ldots\right\} .
$$

Since the state matrix $A$ is assumed to be stable, we have that, given any $\varepsilon>0$ there exists $k_{0}>0$ such that

$$
\left.|| \sum_{k=0}^{\infty} h_{k} e^{-j w k}\right|^{2}-\left|\sum_{k=0}^{k_{0}} h_{k} e^{-j w k}\right|^{2} \mid<\varepsilon, \quad \forall w \in[-\pi, \pi] .
$$

This is equivalent to saying that for $k>k_{0}$ the terms of the impulse response $\left\{h_{k}\right\}$ are negligible i.e., we can effectively assume that system has a fi nite impulse response of length $k_{0}+1$. As a result, the autocorrelation sequence of the impulse response $\left\{h_{k}\right\}_{k=0}^{k_{0}}$ is given by

$$
\begin{aligned}
& \phi_{l}=\sum_{k=0}^{k_{0}-l} h_{k} h_{k+l}, \quad \text { for } 0 \leq l \leq k_{0}, \\
& \phi_{l} \approx 0 \text { for } l>k_{0}, \\
& \phi_{-l}=\phi_{l} .
\end{aligned}
$$

We observe that the $j$-th column of $\bar{\Gamma}$ contains the sequence $\left\{h_{k}\right\}_{k=0}^{k_{0}}$ delayed $j-1$ samples, that is

$$
\bar{\Gamma}=\left[\begin{array}{cccccc}
h_{1} & 0 & \ldots & \ldots & \ldots & 0 \\
\vdots & \ddots & \ddots & & & \vdots \\
h_{k_{0}} & \ldots & h_{1} & 0 & & \vdots \\
0 & \ddots & & \ddots & \ddots & \vdots \\
& \ddots & \ddots & & \ddots & 0 \\
0 & \ldots & 0 & h_{k_{0}} & \ldots & h_{1}
\end{array}\right]
$$

Recall that for the linear model (1) the fi rst element of the impulse response sequence is $h_{0}=0$ since there is no feed-through between the output $y_{k}$ and input $u_{k}$ - see (31). 
Hence, if $N \geq 2 k_{0}+1$ we can write

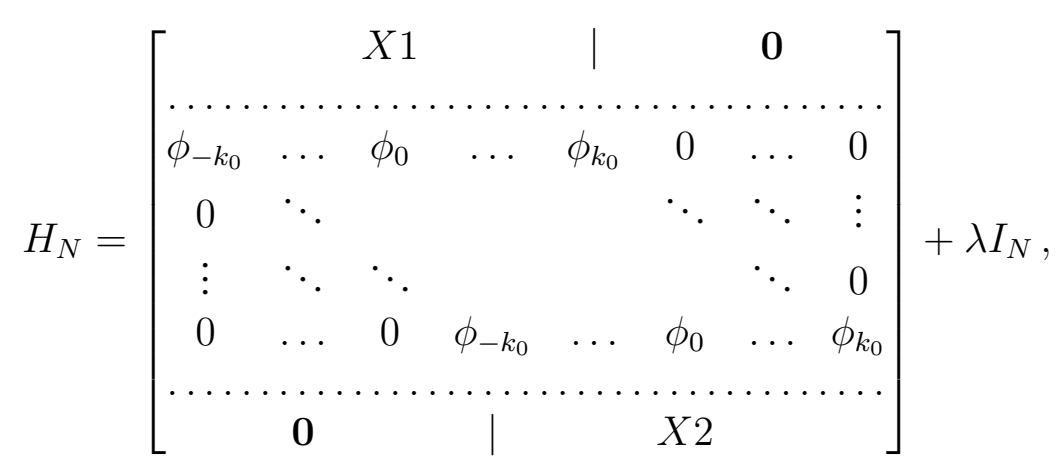

where $X 1$ and $X 2$ are appropriate sub-matrices. Note that we have made use of the notation $H_{N}$ in order to make explicit the dependence on the prediction horizon $N$. We then have the following result.

Theorem 6.1. Consider the discrete time linear system (1) and assume that there exists $k_{0}>0$ such that (32) holds. Let the matrix $H_{N}$ in (34), for $N \geq 2 k_{0}+1$, be the Hessian of the quadratic performance index $J_{N}(x, \mathbf{u})$ in (3) with $\lambda=0$. Let

$$
\Phi(w)=\sum_{l=-\infty}^{\infty} \phi_{l} e^{-j w l}, \quad w \in[-\pi, \pi]
$$

be the Discrete Time Fourier Transform (DTFT) of the autocorrelation sequence in (33). Also, consider the vector

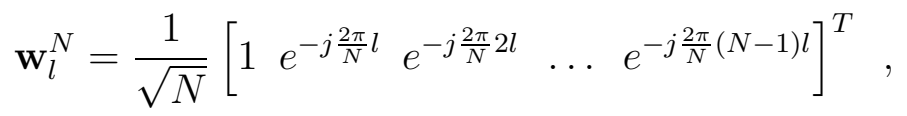

where $l \in \mathbb{Z}$ such that $0 \leq l \leq N-1$ and $\left\|\mathbf{w}_{l}^{N}\right\|_{2}=1$.

Then, for every given frequency $w_{0}=\frac{2 \pi}{N_{0}} l_{0}$, we have that

$$
\lim _{N \rightarrow \infty}\left\|H_{N} \mathbf{w}_{l}^{N}\right\|_{2}=\Phi\left(w_{0}\right)
$$

Proof. We will show that for every $w_{0}=\frac{2 \pi}{N_{0}} l_{0}$ and $\varepsilon_{0}>0$ there exists $L \in \mathbb{Z}^{+}$such that

$$
\left|\left\|H_{N} \mathbf{w}_{l}^{N}\right\|_{2}-\Phi\left(w_{0}\right)\right|<\varepsilon_{0}
$$

whenever $N>L N_{0}$.

We fi rst note that, given $N_{0}>0$ and $0 \leq l_{0} \leq N_{0}-1$, we can obtain, by direct calculation, the following expression.

$$
H_{N_{0}} \mathbf{w}_{l_{0}}^{N_{0}}=\left[\begin{array}{c}
\mathbf{c}_{1} \\
\Phi\left(w_{0}\right) \cdot \mathbf{w}_{l_{0}}^{N_{0}}\left(k_{0}+1: N_{0}-k_{0}\right) \\
\mathbf{c}_{2}
\end{array}\right],
$$


where $\mathbf{c}_{1}$ and $\mathbf{c}_{2}$ are appropriate column vectors of length $k_{0}$ and $\mathbf{w}_{l_{0}}^{N_{0}}\left(k_{0}+1: N_{0}-k_{0}\right)$ is the column vector formed by considering the entries of the vector $\mathbf{w}_{l_{0}}^{N_{0}}$ in (35), starting from $k_{0}+1$ and ending with $N_{0}-k_{0}$.

Subtracting $\Phi\left(w_{0}\right) \mathbf{w}_{l_{0}}^{N_{0}}$ from (36) we obtain

$$
H_{N_{0}} \mathbf{w}_{l_{0}}^{N_{0}}-\Phi\left(w_{0}\right) \mathbf{w}_{l_{0}}^{N_{0}}=\mathbf{d}_{w_{0}}^{N_{0}}
$$

where

$$
\mathbf{d}_{w_{0}}^{N_{0}}=\frac{1}{\sqrt{N_{0}}}\left[\begin{array}{c}
\mathbf{d}_{\mathbf{1}} \\
\mathbf{0}_{N_{0}-2 k_{0}} \\
\mathbf{d}_{\mathbf{2}}
\end{array}\right] .
$$

Both $d_{1}$ and $d_{2}$ are column vectors of the same dimension of vectors $c_{1}$ and $c_{2}$, respectively. Moreover, $\mathbf{0}_{N_{0}-2 k_{0}}$ is a column vector with zero entries and length $N_{0}-2 k_{0}$. It can be easily shown that the norms of both vectors $\mathbf{d}_{1}$ and $\mathbf{d}_{2}$ are bounded. They are determined by the entries of sub-matrices $X 1$ and $X 2$ in (34) (which remain unchanged whenever the prediction horizon $N$ is increased), the fi xed value $\Phi\left(u_{0}\right)$ and the entries of vector $\mathbf{w}_{l_{0}}^{N_{0}}$ which are bounded. As a result, we can find $T_{w_{0}}>0$ such that

$$
\left\|\mathbf{d}_{w_{0}}^{N_{0}}\right\|_{2}=\frac{1}{\sqrt{N_{0}}} \sqrt{\left\|\mathbf{d}_{1}\right\|_{2}^{2}+\left\|\mathbf{d}_{2}\right\|_{2}^{2}} \leq \frac{1}{\sqrt{N_{0}}} T_{w_{0}}
$$

We now choose $N=L N_{0}$ and $l=L l_{0}$, for some $L \in \mathbb{Z}^{+}$, such that

$$
\frac{2 \pi l}{N}=\frac{2 \pi l_{0}}{N_{0}}=w_{0}
$$

As a result, the following holds.

$$
\begin{aligned}
& \left|\left\|H_{N} \mathbf{w}_{l}^{N}\right\|_{2}-\Phi\left(w_{0}\right)\right|=\left|\left\|H_{N} \mathbf{w}_{l}^{N}\right\|_{2}-\Phi\left(w_{0}\right)\left\|\mathbf{w}_{l}^{N}\right\|_{2}\right| \\
& \leq\left\|H_{N} \mathbf{w}_{l}^{N}-\Phi\left(w_{0}\right) \mathbf{w}_{l}^{N}\right\|_{2}=\left\|\mathbf{d}_{w_{0}}^{N}\right\|_{2} .
\end{aligned}
$$

This allows us to conclude that for every $\varepsilon_{0}>0$

$$
\left\|\mathbf{d}_{w_{0}}^{N}\right\|_{2}<\varepsilon_{0} \Longrightarrow\left|\left\|H_{N} \mathbf{w}_{l}^{N}\right\|_{2}-\Phi\left(w_{0}\right)\right|<\varepsilon_{0} .
$$

Using the bound derived in (37), we now can fi nd $L>0$ such that

$$
\left\|\mathbf{d}_{w_{0}}^{N}\right\|_{2} \leq \frac{1}{\sqrt{N}} T_{w_{0}}=\frac{1}{\sqrt{L N_{0}}} T_{w_{0}}<\varepsilon_{0} .
$$

The above is satisfi ed for $L \in \mathbb{Z}^{+}$such that

$$
L>\frac{T_{w_{0}}^{2}}{N_{0} \varepsilon_{0}^{2}} .
$$

This completes the Proof. 
Corollary 6.2. Consider the same conditions of Theorem 6.1 and choose $R=\lambda>0$. Then

$$
\lim _{N \rightarrow \infty}\left\|H_{N} \mathbf{w}_{l}^{N}\right\|_{2}=\Phi\left(w_{0}\right)+\lambda
$$

Proof. Directly from Theorem 6.1.

Recall that $H_{N}$ is symmetric and positive defi nite, so that any singular value of $H_{N}$ is equal to a corresponding eigenvalue. As a result, Theorem 6.1 shows that given any frequency $w_{0} \in[-\pi, \pi]$, we can find a prediction horizon $N$ such that there exists at least one singular value of the Hessian $H_{N}$ that is arbitrarily close to the energy density spectrum $\Phi(\cdot)$ of system (1) evaluated at $w=w_{0}$. The importance of this result is twofold. It shows that for long prediction horizons $N$ there will be, in general, several singular values close to zero, since they correspond to high frequency components of $\Phi(w)$. In addition, Theorem 6.1 shows that, for long horizons $N$, one can avoid the need to compute the singular values by simply working in the frequency domain.

The following example illustrates the result.

Example 6.1. Consider a discrete-time resonant SISO system with state space matrices defi ned as follows:

$$
A=\left[\begin{array}{cc}
1.5293 & -0.7408 \\
1 & 0
\end{array}\right], B=\left[\begin{array}{c}
0.5 \\
0
\end{array}\right], C=\left[\begin{array}{ll}
0.2222 & 0.2009
\end{array}\right]
$$

Consider the fi nite horizon optimal control problem $\mathscr{P}_{N}(x)$ with performance index (3) and $Q=C^{T} C, P=Q$ and $R=0$.

Fig. 2 compares the singular values of the Hessian $H_{N}$ (circles), for two different prediction horizons, with the energy density spectrum $\Phi(w)$ (continuous line) of the system in (38). We observe that as the prediction horizon $N$ increases, from $N=51$ to $N=401$, the singular values of the Hessian converge, in a well defi ned sense, to the magnitude of $\Phi(w)$.

\section{Simulation example}

We illustrate the application of the SVD receding horizon control strategy described in Section 4 to a 3 input - 3 output MIMO plant. Consider a continuous time stable system defi ned by the following transfer function matrix:

$$
G(s)=\left[\begin{array}{ccc}
\frac{1}{s^{2}+0.6 s+1} & \frac{4}{s+4} & \frac{-2 s+1}{s^{2}+2.5 s+1} \\
\frac{-4 s+2}{s^{2}+3 s+2} & \frac{1}{s^{2}+0.6 s+1} & \frac{1}{s+1} \\
\frac{2 s+4}{s^{2}+4 s+4} & \frac{0.5}{s+0.5} & \frac{1.6}{s^{2}+0.64 s+1.6}
\end{array}\right] .
$$

Assume that $G(s)$ is appropriately discretized with a sampling period $T_{s}=0.2[s]$. Consider

$$
Q=I_{n}, \quad R=0.1 I_{m}, \text { and } N=15
$$




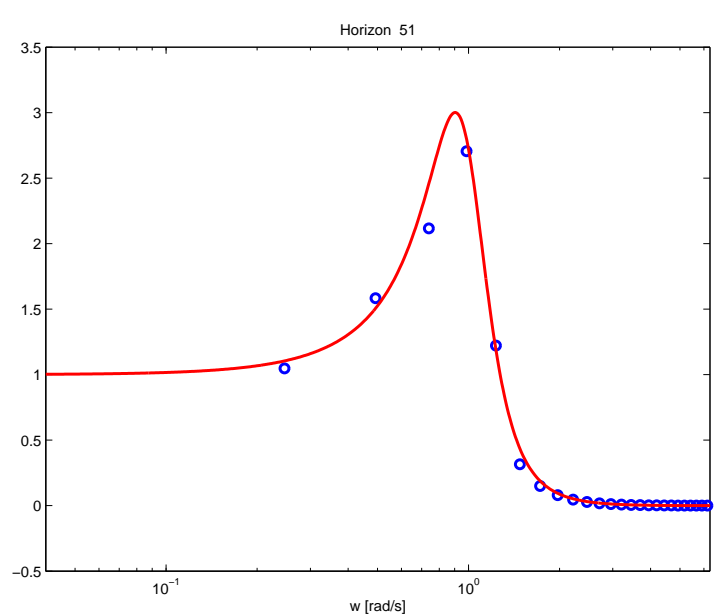

(a) $N=51$

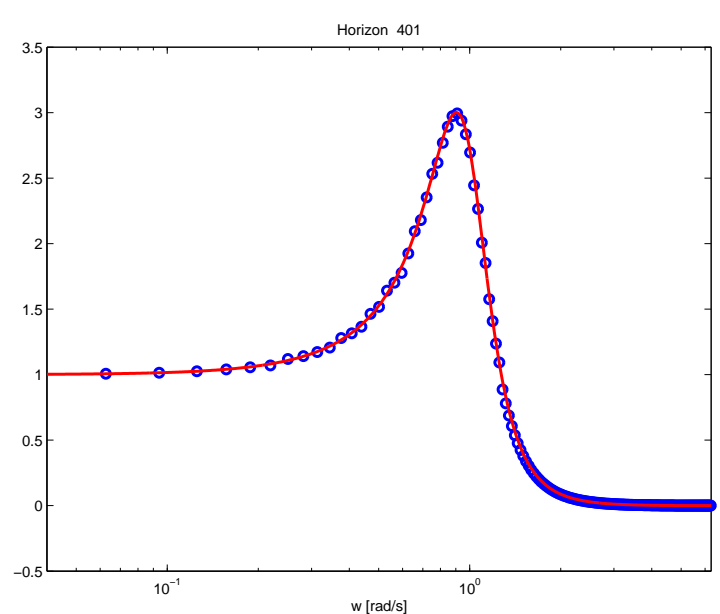

(b) $N=401$.

Figure 2: Singular values of the Hessian $H_{N}$ (circles) of Example 6.1 for two different prediction horizons. The continuous line represents the energy density spectrum $\Phi(w)$ of system (38).

Also, assume that each of the inputs to the plant (7) is constrained to the interval $[-1,1]$.

Fig. 3 shows the singular values of the Hessian $H$. We see that, in this case, $H$ is poorly conditioned. In fact, several of the singular values of $H$ are close to zero (they converge to 0.1 - see Corollary 6.2) whilst the first six singular values are those that dominate the performance index. Based on the discussion in Section 6, we expect that the SVD- RHC strategy will perform adequately in this case.

We compare the response of the closed loop system using MPC and using the proposed SVD- RHC strategy for a given initial condition. Fig. 4 shows the time evolution of the outputs of the plant using both strategies. We observe that the results are similar, despite the fact that no solution to the QP problem was required by the SVD- RHC strategy. Fig. 5 shows the time evolution of the inputs. Although being somewhat dissimilar to the full MPC result, the control signals generated by the proposed strategy incorporate the components that affect the reduction of the performance index the most. Note that the 3 inputs to the plant hit the constraints during several samples, indicating that the proposed SVD- RHC strategy can be effective even in the presence of tight constraints.

We fi nally show in Fig. 6 the time evolution of the parameter $\gamma$ which defi nes the number of elements in the basis function expansion of $\mathbf{u}_{u c}^{o}(x)$ being used by the SVDRHC strategy. We observe that during the initial transient response, the strategy discards several of the elements of $\widetilde{\mathbf{u}}_{u c}^{o}(x)$. For example, note that less than 6 components are used during the first $1[s]$ of the simulation.

Finally, the stability result of Theorem 5.3 ensures that the closed loop is exponentially stable if

$$
\gamma \geq \gamma^{*}=36.4 \text { with } \delta=0.001 \text {. }
$$




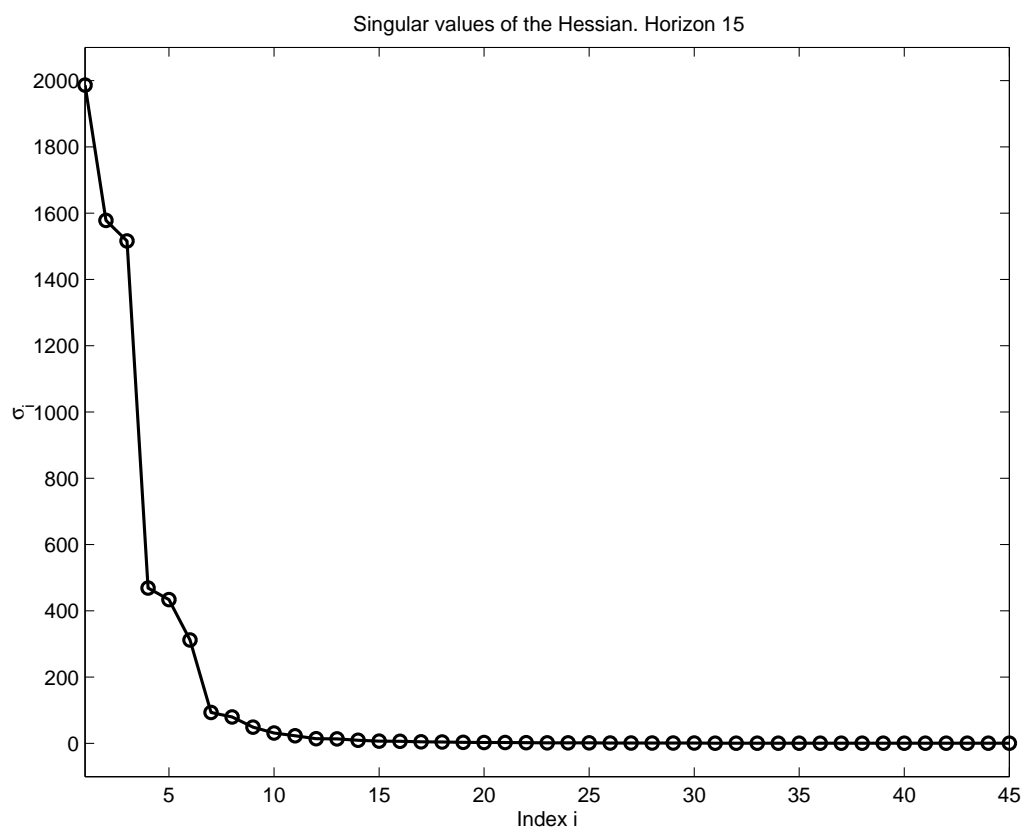

Figure 3: Singular values of matrix $H$ with horizon $N=15$.
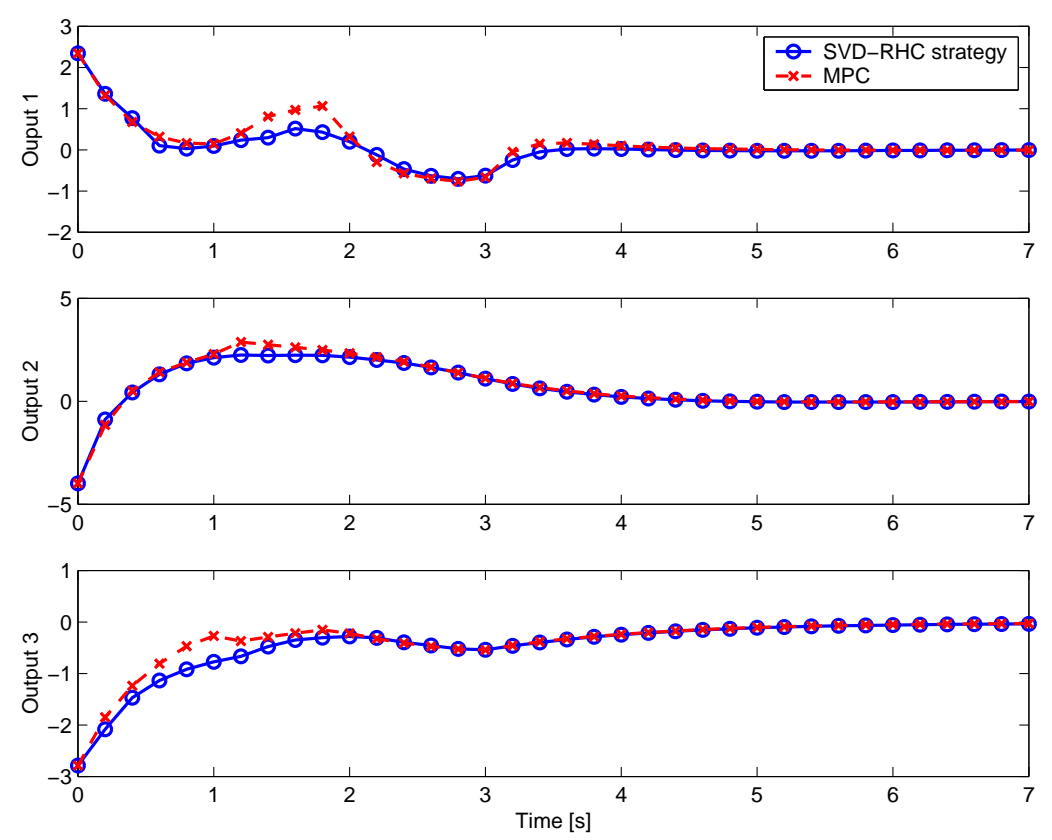

Figure 4: Comparison between MPC (stars and dashed line) and the SVD- RHC strategy (circles and continuous line): Output response. 

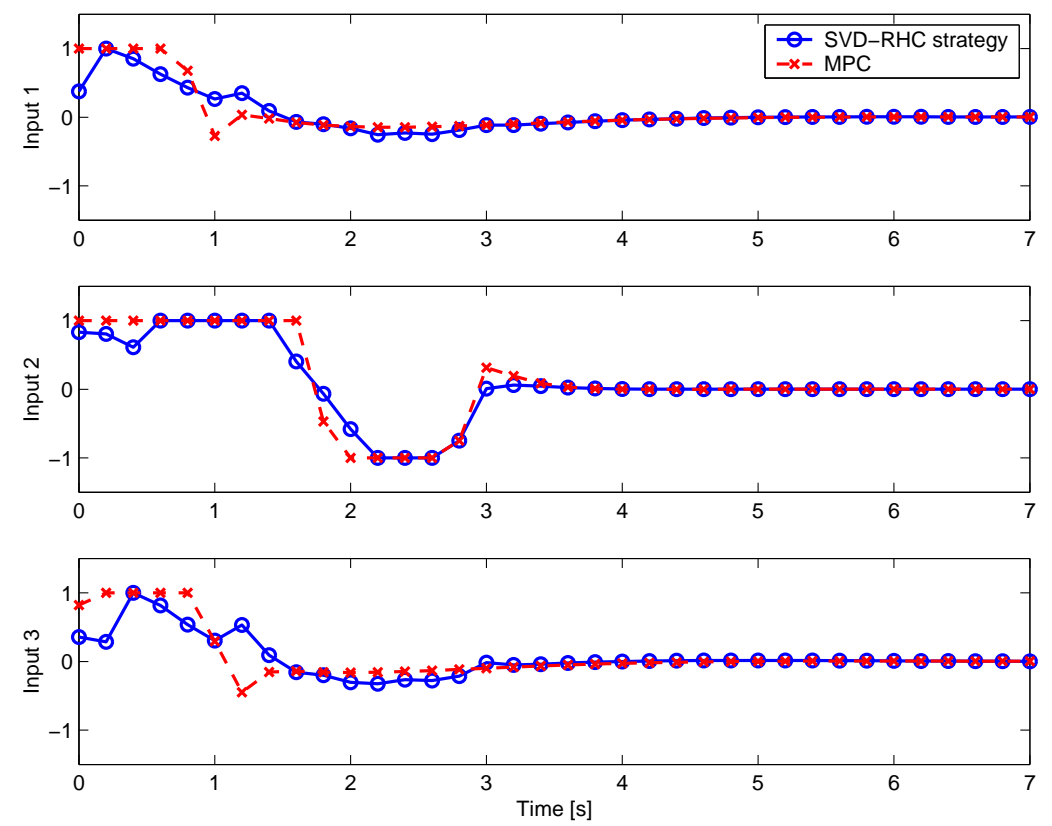

Figure 5: Comparison between MPC (stars and dashed line) and the SVD- RHC strategy response (circles and continuous line): Input response.

However, this is just a suffi cient condition for stability. Indeed, the simulation presented in this Section clearly shows that the closed loop is stable despite the fact that condition (39) is not satisfi ed.

\section{Conclusions}

We have proposed a receding horizon control strategy for input constrained linear systems based on a sub-optimal solution to the fi nite horizon optimal control problem solved at each time step. The sub-optimal solution is constructed from a basis function expansion of the unconstrained solution to the optimization problem. At each sampling time, the proposed strategy determines a feasible control sequence by selecting a variable subset of the basis representation. For systems in which the Hessian of the performance index has large condition number, the performance obtained using the proposed strategy is comparable to the performance obtained by solving the full on-line optimization problem. We have also shown that, for prediction horizons long enough, the singular values of the Hessian are arbitrarily close to the magnitude of the energy density spectrum of the system seen by the performance index. 


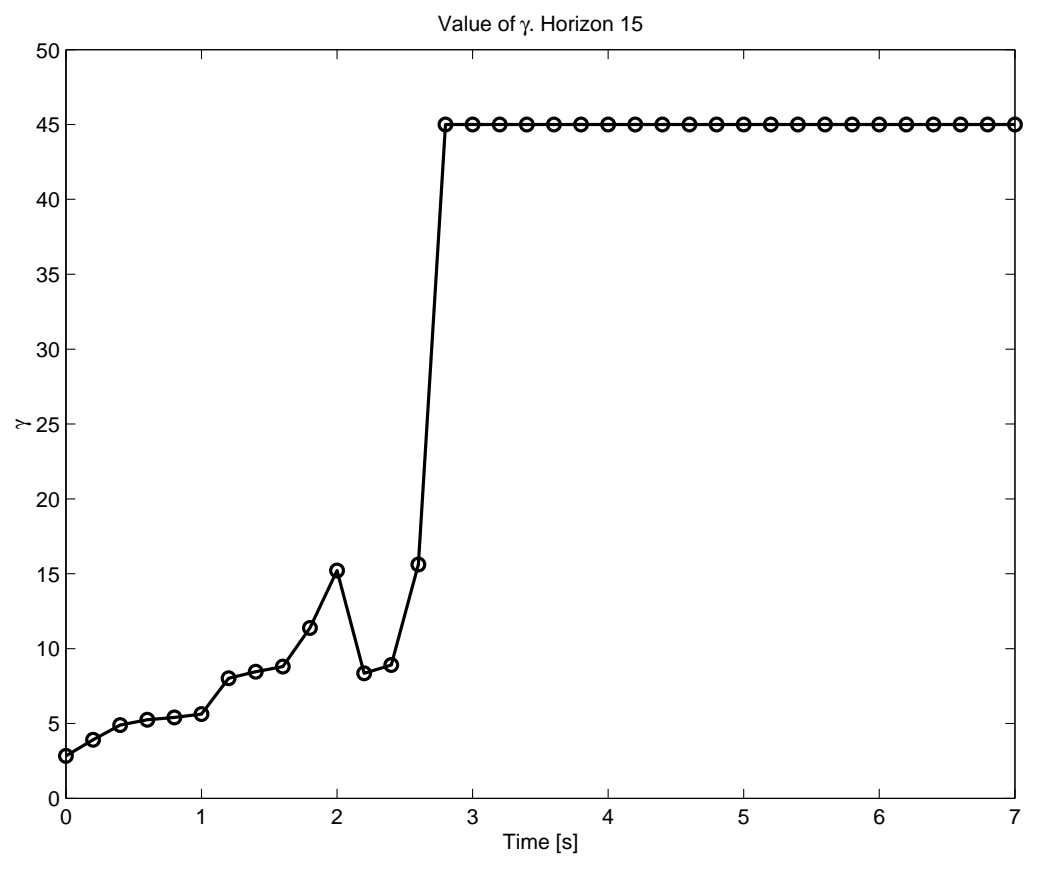

Figure 6: Number of components considered by the SVD- RHC strategy at each time step as represented by $\gamma \triangleq r+\alpha$.

\section{Acknowledgments}

The authors would like to acknowledge the valuable comments and suggestions received from the reviewers of this manuscript.

\section{References}

Aoyama, Atsushi, Geoffrey Bell and Stephen P. Walsh (1997). Implementation issues in quadratic model predictive control. In: Proceedings of the American Control Conference. Albuquerque, New Mexico, USA.. pp. 3375-3379.

Bartlett, Roscoe A., Lorenz T. Biegler, Johan Backstrom and Vipin Gopal (2002). Quadratic programming algorithms for large-scale model predictive control. Journal of Process Control 12(7), 775-795.

Bemporad, Alberto, Manfred Morari, Vivek Dua and Efstratios N. Pistikopoulos (2002). The explicit linear quadratic regulator for constrained systems. Automatica 38, 3-20.

Blanchini, Franco (1999). Set invariance in control. Automatica 35, 1747-1767.

Featherstone, Andrew P., Jeremy G. VanAntwerp and Richard D. Braatz (2000). Identification and control of sheet and film processes. Springer Verlag. London. 
Golub, Gene H. and Charles F. Van Loan (1996). Matrix computations. 3rd ed.. The Johns Hopkins University Press. Baltimore, Maryland, USA.

Johansen, Tor A., Idar Petersen and Olav Slupphaug (2002). Explicit sub-optimal linear quadratic regulation with state and input constraints. Automatica 38, 1099-1111.

Kojima, Akira and Manfred Morari (2001). LQ control for constrained continuous-time systems: an approach based on singular value decomposition. In: Proceedings of the 40th IEEE Conference on Decision and Control. Orlando, Florida USA.

Kouvaritakis, B., M. Cannon and J. A. Rossiter (2002). Who needs QP for linear MPC anyway?. Automatica 38, 879-884.

Mayne, D. Q., J. B. Rawlings, C. V. Rao and P. O. M. Scokaert (2000). Constrained model predictive control: stability and optimality. Automatica 36(6), 789-814.

Qin, S. Joe and T. A. Badgwell (2003). A survey of industrial model predictive control technology. Control Engineering Practice 11, 733-764.

Rao, C. V., S. J. Wright and J. B. Rawlings (1998). Application of interior-point methods to model predictive control. Journal of Optimization Theory and Applications 99(3), 723-757.

Rojas, O. J., G. C. Goodwin and G. V. Johnston (2002a). Spatial frequency anti-windup strategy for cross directional control problems. IEE Proceedings Control Theory and Applications 149(5), 414-422. Submitted June 2001.

Rojas, Osvaldo J. and Graham C. Goodwin (2003). On the asymptotic properties of the Hessian in discrete-time linear quadratic control. Technical report. School of Electrical Eng. \& Computer Sc. , The University of Newcastle.

Rojas, Osvaldo J., Graham C. Goodwin and André Desbiens (2002b). Study of an adaptive anti-windup strategy for cross-directional control systems. In: Proceedings of the 41st IEEE Conference on Decision and Control. Las Vegas, Nevada, USA.

Rojas, Osvaldo J., Graham C. Goodwin, Arie Feuer and María M. Serón (2003). A suboptimal receding horizon control strategy for constrained linear systems. In: Proceedings of the American Control Conference. Denver, Colorado, USA.

Rossiter, J. A., B. Kouvaritakis and M. J. Rice (1998). A numerically robust state-space approach to stable-predictive control strategies. Automatica 34(1), 65-73.

Sanchis, J., C. Ramos, M. Martínez and X. Blasco (2001). Principal component weighting (PCW) for constrained GPC design. In: Proceedings of 9th Mediterranean Conference on Control and Automation. Dubrovnik, Croatia.

Serón, María M., José A. De Doná and Graham C. Goodwin (2000). Global analytical model predictive control with input constraints. In: Proceedings of the 39th IEEE Conference on Decision and Control. Sydney, Australia. pp. 154-159. 